\title{
Improving quality of care in nursing homes: What works?
}

\author{
Robin L. Stadnyk PhD BScOT, Heidi Lauckner PhD BScOT, Barry Clarke MD COE \\ See related research article by Boorsma and colleagues at www.cmaj.ca/lookup/doi/10.1503/cmaj.101498 and synopsis on page 1262 .
}

Competing interests: None declared.

This article was solicited and has not been peer reviewed.

Correspondence to: Robin L. Stadnyk, rstadnyk@dal.ca

CMAJ 2011. DOI:10.1503 /cmaj.110789
$\mathrm{T}$ he research of Boorsma and colleagues, published in this issue of CMAJ, is notable for framing its approach to intervention in a multidisciplinary chronic disease model of care. This is an important contribution to improving care in nursing homes because it integrates process measures with outcomes that are important to the resident's functional status and quality of life. In contrast, much of the North American research on care in nursing homes focuses on effectiveness, improving quality or the culture of care. These three foci result in fundamental differences in the reported outcomes of interest. In a discussion of "what works" in terms of the care provided in nursing homes, the first question might be "What are we trying to change?"

For the purposes of this commentary, nursing home care refers to type II care as defined by the Federal-Provincial Working Party on Patient Care Classification ${ }^{2}$ (i.e., medical supervision and supervision by professional nurses, assistance with activities of daily living and personal care on a 24-hour basis and provisions to meet psychosocial needs). This may be a higher level of care than was provided by the facilities studied by Boorsma and colleagues. ${ }^{1}$

Like other health services used by older adults, care in nursing homes has garnered attention owing to the aging demographic in North America and elsewhere. There are two competing pressures resulting from this demographic shift. The first is to ensure that resources are used as efficiently as possible while providing good outcomes of care. This has resulted in a push for research that examines the effectiveness of interventions and improvements in quality. The second is for a culture of care that is more resident-directed and homelike,

\section{KEY POINTS}

- The focus of nursing home research - effectiveness, quality improvement or culture of care - influences the outcomes of care examined.

- Research on the effectiveness and quality of care is plentiful and often includes randomized controlled trials, but research on the culture of care and staff empowerment is lacking and not as rigorous.

- Models of care for chronic disease are not widely used in nursing homes, but they show promise as a strategy to improve processes and outcomes of care. recognizing that nursing homes are places where people both live and receive care.

\section{Key areas of research}

Research on care provided by nursing homes is further complicated because the health status of residents in these facilities does not improve in the dramatic ways seen in acute care. Adopting the chronic disease model of care proposed by Boorsma and colleagues ${ }^{1}$ appropriately frames the chronic nature of health conditions affecting many older adults.

\section{Effectiveness of treatment}

Much research including clinical trials has examined the effectiveness of particular approaches to treatment in nursing homes, covering areas such as skin care and integrity, behaviour management, pain management and incontinence. Often, the results of such research show that "what works" involves simple, low-technology solutions that may increase staff time. One review of 14 clinical trials of interventions to reduce incontinence among residents of nursing homes ${ }^{3}$ showed that, among residents with urinary incontinence, prompted voiding alone and prompted voiding with exercise were associated with modest short-term improvement of the condition; medications provided only small additional benefit when used with prompted voiding. Similarly, a randomized controlled trial of methods to improve residents' caloric consumption showed that offering them snacks and assistance was more effective than offering them supplements. ${ }^{4}$

\section{Quality improvement}

High-quality research also abounds in the area of quality improvement, often focusing on process measures such as compliance with particular protocols (e.g., education, audit-feedback and consultation and coaching with designated staff). Outcomes such as the health status of residents may or may not be reported, and there are relatively few examples of nursing homes assessing and monitoring improvements in quality with tools such as the Resident Assessment Indicators. ${ }^{5}$ Most studies show that programs to improve quality were successful.

All editorial matter in CMAJ represents the opinions of the authors and not necessarily those of the Canadian Medical Association. 
Lack of staff time or staff turnover were cited as the most frequent reasons for unsuccessful initiatives. ${ }^{5}$

\section{Culture of care}

There is a paucity of research exploring the changed culture of care within nursing homes, although there has been rapid adoption of models associated with these changes. ${ }^{6}$ For example, the Eden Alternative, used in many Canadian settings, aims to eliminate boredom, loneliness and helplessness by applying 10 principles that include meaningful activity, stimulating environments, social relationships and decision-making by elders or those closest to them. Research looking at changes in culture typically values outcomes of resident-centred care, satisfaction and quality of life. In a review of such research, Mueller ${ }^{7}$ notes that most available research is descriptive and case-based; however, three quasiexperimental studies showed positive improvements among residents and staff.

\section{Staff empowerment}

Approaches that consider culture of care, chronic disease and, increasingly, quality improvement emphasize empowering the staff who provide direct care to solve problems and make decisions with residents. Related to the empowerment of frontline staff are team approaches to care that emphasize communication between providers of care. ${ }^{8}$ Research on staff empowerment is still evolving. Using a nonequivalent control, pretest posttest design, Yeatts and Cready ${ }^{9}$ showed that empowered teams had a positive impact on the performance of nursing assistants and the families' perceptions of responsiveness to residents' preferences. Other research has shown that empowered staff contribute to an improved quality of life for residents. ${ }^{10}$ Promising Canadian research (i.e., the Safer Healthcare Now projects) has shown a positive correlation between staff empowerment and quality improvement in settings that provide acute care; future research in British Columbia and Alberta will look at how well this approach translates to nursing homes. ${ }^{11}$

Wagner's model of chronic care ${ }^{12}$ considers the roles of the community, the health system and informed, active patients and care providers in the management of chronic disease. Outcomes of interest include the health and functional status of clients, as well as the empowerment of clients and care providers. Although there is little published research on chronic disease models of care in nursing homes, more use of these models is likely given the good fit between them and the changing culture of care. For example, an emerging model of care in Nova Scotia, Care by Design, started with assigning one community physician per nursing home floor who participated in regular weekly meetings with staff. ${ }^{13}$ The model is expanding to include multidisciplinary teams, management of chronic disease and principles of end-of-life care. In July 2011, a research project using a mixedmethod approach will examine process measures and pre- and postimplementation outcomes including admissions to hospital, deaths in hospital, wounds, falls and rates of polypharmacy (Barry Clarke, written communication to Robin Stadnyk, 2011 May 15).

In research on the quality of care in nursing homes, a chronic disease model that emphasizes multidisciplinary coordinated care and empowering approaches may help integrate the consideration of care processes with the physical, functional and psychosocial dimensions of the lives of residents. Such models show promise in determining "what works" in these settings.

\section{References}

1. Boorsma M, Fritjers DHM, Knol DL, et al. Effects of multidisciplinary integrated care on quality of care in residential care facilities for elderly people: a cluster randomized trial. CMAJ 2011;183:E724-32.

2. Federal-Provincial Working Party on Patient Classification. Report: types of care. Ottawa (ON): The Party; 1973.

3. Fink HA, Taylor BC, Tacklind JW, et al. Treatment interventions in nursing home residents with urinary incontinence: a systematic review of randomized trials. Mayo Clin Proc 2008;83:1332-43.

4. Simmons SF, Keeler E, Zhuo X, et al. Prevention of unintentional weight loss in nursing home residents: a controlled trial of feeding assistance. J Am Geriatr Soc 2008;56:1466-73.

5. Sales AE, Bostrom AM, Bucknall T, et al. The use of data for process and quality improvement in long-term care and home care: a systematic review of the literature. J Am Med Dir Assoc 2011; Feb. 15. [Epub ahead of print].

6. Rahman AN, Schnelle JF. The nursing home culture-change movement: recent past, present, and future directions for research. Gerontologist 2008;48:142-8.

7. Mueller C. Research in culture change in nursing homes. Proceedings of the Nurses Involvement In Culture Change; 2008 Oct. 27 28; New York (NY). Available: http://hartfordign.org/uploads/File /issue_culture_change/Culture_Change_Background_Mueller.pdf (accessed 2011 May 20).

8. Crotty M, Halbert J, Rowett D, et al. An outreach geriatric medication advisory service in residential aged care: a randomised controlled trial of case conferencing. Age Ageing 2004;33:612-7.

9. Yeatts DE, Cready CM. Consequences of empowered CNA teams in nursing home settings: a longitudinal assessment. Gerontologist 2007;47:323-39.

10. Bishop CE, Weinberg DB, Leutz W, et al. M. Zincavage R. Nursing assistants' job commitment: effect of nursing home organizational factors and impact on resident well-being. Gerontologist 2008;48(Suppl 1):36-45.

11. KUSP Knowledge Utilization Studies Program. Safer care for older persons (in residential) environments SCOPE. Edmonton (AB): University of Alberta; 2010. Available:www.kusp.ualberta.ca /Research/ActiveProjects/SCOPE.aspx (accessed 2011 May 20).

12. For the record. CMAJ 2009;181:E239-41.

13. Wagner EH. Chronic disease management: what will it take to improve care for chronic illness? Eff Clin Pract 1998;1:2-4.

Affiliations: From the School of Occupational Therapy (Stadnyk, Lauckner), the Department of Family Medicine (Clarke), Dalhousie University, Halifax NS; and Capital Health (Clarke), Halifax, NS

Contributors: Robin Stadnyk wrote the first draft of the paper and incorporated feedback from coauthors in subsequent drafts. Heidi Lauckner provided background literature and provided structural and substantive edits to drafts of the article. Barry Clarke critically reviewed the first draft and added content to the article. All of the authors approved the final version submitted for publication. 